\title{
AN APPLICATION OF SCHLÄFLI'S MODULAR EQUATION TO A CONJECTURE OF RAMANUJAN $\dagger$
}

\author{
D. H. LEHMER
}

In 1918 Ramanujan $\ddagger$ made the following conjecture:

If $q=5,7$, or 11 , and if $24 n-1$ is divisible by $q^{\alpha}$, then the number $p(n)$ of unrestricted partitions of $n$ is divisible by $q^{\alpha}$.

Ramanujan himself proved this conjecture to be true in case $\ddagger$ $q^{\alpha}=5,7,5^{2}$, and $7^{2}$, and also $\S$ for $q^{\alpha}=11$ and $11^{2}$. It has since been proved $\|$ for $q^{\alpha}=5^{3}$. Some modification of the conjecture is necessary, however, since, as Chowla $\uparrow$ was first to notice, it fails for $q^{\alpha}=7^{3}$. In fact, since $24 \cdot 243-1=5831$ is divisible by $7^{3}$, it would follow from the conjecture that $p(243)$ is also divisible by $7^{3}$. However, Gupta's table** of $p(n)$ gives

$$
p(243)=133978259344888,
$$

a number $\dagger \dagger$ which is not divisible by $7^{3}$. It occurred to the writer that it would be worth while making an investigation of $p(599)$ and $p(721)$ relative to their divisibility by $5^{4}$ and $11^{3}$ respectively. $\ddagger$ To obtain the value of $p(n)$ for these isolated values of $n$ beyond the limits of then existing tables, use was made of the celebrated Hardy-Ramanujan series, $\$ \S$ which may be written

$$
p(n)=\frac{(12)^{1 / 2}}{\mu(24 n-1)} \sum_{k=1}^{N} A_{k}^{*}(n)(\mu-k) e^{\mu / k}+r_{n}(N),
$$

where we have written $\mu$ for $\pi(24 n-1)^{1 / 2} / 6$. By taking $N=18$ for $n=599$, and $N=21$ for $n=721$, values were obtained for the series in

$\dagger$ Presented to the Society, September 10, 1937.

¥ Proceedings of the London Mathematical Society, vol. 19 (1919), pp. 207-210; Collected Papers, pp. 210-213.

$\S$ Mathematische Zeitschrift, vol. 9 (1921), pp. 147-153; Collected Papers, pp. 232-238. A proof for $11^{2}$ is in one of his notebooks.

\| See Bulletin of the Academy of Sciences, U.R.S.S., 1933, ro. 6, pp. 763-800.

I Journal of the London Mathematical Society, vol. 9 (1934), p. 247.

** Proceedings of the London Mathematical Society, (2), vol. 39 (1935), p. 149.

$\dagger \dagger$ This number has been verified by the present writer.

$\ddagger$ Journal of the London Mathematical Society, vol. 11 (1936), pp. 114-118.

$\S \S$ Proceedings of the London Mathematical Society, (2), vol. 17 (1918), pp. 75115. Ramanujan's Collected Papers, pp. 276-309. 
(1) which differed from integers by only .00027 and .00041 . Moreover, these integers were divisible by $5^{4}$ and $11^{3}$ as predicted by the conjecture. Still it could not be concluded that these integers were actually $p(599)$ and $p(721)$ since nothing was known about $r_{n}(N)$ at that time beyond the result of Hardy and Ramanujan that, if $\alpha>0$,

$$
r_{n}\left(\alpha n^{1 / 2}\right)=O\left(n^{-1 / 4}\right) .
$$

This uncertainty has since been removed in two ways. In the first place Gupta $\dagger$ has extended his table of $p(n)$ to $p(600)$ and finds the writer's value for $p(599)$; he confirms $p(721)$ with respect to the modulus 247 . On the other hand, Rademacher $\$$ has recently proved that if $(\mu-k) e^{\mu / k}$ is replaced by $(\mu-k) e^{\mu / k}+(\mu+k) e^{-\mu / k}$ in (1), we obtain a convergent series for $p(n)$. With this important fact established it is possible to give an estimate for the remainder $r_{n}(N)$ and thus the application of the Hardy-Ramanujan series for any particular $n$ is put on a firm basis.

The application of (1) for large values of $n$ was now beset with a further difficulty, namely, that of evaluating the coefficients $A_{k}^{*}(n)$. These numbers are usually written $A_{k}(n) / k^{1 / 2}$, where the $A_{k}(n)$ are complicated sums of $24 k$ th roots of unity arising from the theory of elliptic modular functions. The approximate values of $A_{k}(n)$ for all $n$ and for $k \leqq 20$ have been tabulated $\S$ by the writer. For large $n$, however, the series (1) demands more accuracy in the first few $A^{*}$ 's, and of course additional $A^{*}$ 's. To meet this problem the writer has made a special investigation $\|$ of $A_{k}(n)$, and has obtained the unexpected result that $A_{k}^{*}(n)$ is merely a power of 2 times a cosine of a rational multiple of $\pi$. As a consequence, if $k$ is small, $A_{k}(n)$ is a root of a well known algebraic equation of low degree with integer coefficients, so that $A_{k}{ }^{*}$ may be easily found with the high degree of accuracy demanded of the first few $A^{*}$ 's, while if $k$ is large, $A_{k}^{*}(n)$ may be obtained from any standard table of natural cosines.

Another consequence is of importance to the estimation of the remainder $r_{n}(N)$. In fact $A_{k}^{*}(n)$ turns out to be much smaller than might be expected. A priori one has as a trivial estimate

$\dagger$ Proceedings of the Indian Academy of Sciences, vol. 4 (1936), pp. 625-629. His extended table is in Proceedings of the London Mathematical Society, vol. 42 (1937), pp. 547-549. 254.

$\ddagger$ Proceedings of the London Mathematical Society, (2), vol. 43 (1937), pp. 241-

§ Journal of the London Mathematical Society, vol. 11 (1936), pp. 117-118. (Erratum. For $A_{20}(n)$ read $A_{20}(n+5)$ ).)

\| To appear in the Transactions of this Society. 


$$
\left|A_{k}^{*}(n)\right| \leqq k^{1 / 2} \text {. }
$$

One may now prove for example that $\dagger$

$$
\left|A_{k}^{*}(n)\right|<2 k^{1 / 3} \text {. }
$$

Using this estimate one may prove that

$$
\left|r_{n}(N)\right|<\frac{\pi^{2} N^{7 / 3}}{3^{1 / 2} \mu^{3}}\left\{\sinh \frac{\mu}{N}+\frac{\mu^{3}}{6 N^{3}}+\left(1+\frac{N}{\mu}\right)\left(\frac{1}{7}+\frac{1}{3} \mu^{1 / 3} N^{-5 / 3}\right)\right\},
$$

a result which is by no means the best possible.

In the application of the Hardy-Ramanujan series there remains only the difficulty of computing $e^{\mu}$. Even when $\mu$ is only moderately large the use of the series for $e^{\mu}$ is too laborious and risky to be recommended. If $\mu$ is not too large, it is possible to evaluate $e^{\mu}$ by means of tables of very accurate logarithms such as Wolfram's 48 figure natural logarithms or Sharp's 61 figure common logarithms. $\ddagger$ This may be done by finding, by continued fractions, an approximation $E$ to $e^{\mu}$ so that

$$
e^{\mu}=E(1 \pm \epsilon)
$$

and such that $\log E$ is a simple combination of tabulated logarithms. Thence

$$
\log (1 \pm \epsilon)=\mu-\log E= \pm \delta
$$

may be found and will be quite small if $E$ is a good enough approximation. It follows that

$$
e^{\mu}=E e^{ \pm \delta}=E\left(1 \pm \delta+\frac{\delta^{2}}{2 !} \pm \frac{\delta^{3}}{3 !}+\cdots\right)
$$

where the series converges rapidly. This method of computation, which frequently involves the use of a factor table, was a favorite with Gauss. $\$$

An entirely different method of computing $e^{\mu}$ is afforded by the

$\dagger$ Or indeed even better results than $A_{k}^{*}(n)=O\left(n^{\mathrm{e}}\right)$.

$\ddagger$ These tables are reprinted in several places such as Callet's Tables Portatives, or Peter's Zehnstellige Logarithmen. For complete information see Henderson, Bibliotheca Tabularum Mathematicarum, vol. 1, part A (Tracts for Computers, no. 13), Cambridge, 1926, pp. 195, 204.

$\S$ See for instance his Werke, III, pp. 426-432. The fact that many typographical errors exist in the several reprints of the above mentioned tables of logarithms makes it desirable to use two different values of $E$ and thus to make two independent calculations. 
theory of elliptic modular functions. The fact that these functions are of practical use in evaluating special exponentials of the type $\exp \left[\pi D^{1 / 2}\right]$ appears to have been noticed first by Hermite $\dagger$ and later by Kronecker. $\ddagger$ This same fact was rediscovered by Ramanujan $\S$ in an early paper. In the present paper we give a modification of this method to the special problem at hand and give the results of applying it to the calculation of $p(n)$ for $n=1224,2052,2474$, and 14031 .

In this method we use to the fullest advantage the fact that $\mu=\pi(24 n-1)^{1 / 2} / 6$ is of the form $\pi m \nu^{1 / 2} / 6$ where $m$ is a power of 5,7 , or 11 , in view of the hypothesis of Ramanujan's conjecture.

In the notation of Weber, $\|$ the class-invariants $f$ and $f_{1}$ are defined by

$$
\begin{aligned}
& f\left((-D)^{1 / 2}\right)=\exp \left[\pi D^{1 / 2} / 24\right] \prod_{k=1}^{\infty}\left(1+\exp \left[-(2 k-1) \pi D^{1 / 2}\right]\right) \\
& f_{1}\left((-D)^{1 / 2}\right)=\exp \left[\pi D^{1 / 2} / 24\right] \prod_{k=1}^{\infty}\left(1-\exp \left[-(2 k-1) \pi D^{1 / 2}\right]\right)
\end{aligned}
$$

Each class-invariant is the root of an algebraic equation with integer coefficients solvable in radicals. The feature of this method is the exploitation of the algebraic character of $f$ and $f_{1}$. The derivation of the equation for $f$ or $f_{1}$ in case $D$ is large and arbitrary is a difficult matter. In case the number of classes of binary quadratic forms of determinant $-D$ is fairly small, however, the equations can be found rather easily, and in many cases the expressions for $f$ and $f_{1}$ in radicals

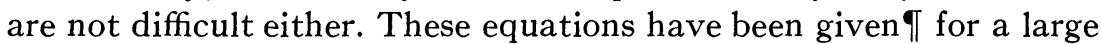
number of values of $D$. In case $D$ contains a square factor $D=s^{2} D^{\prime}$, it is particularly simple to derive the equations for $f\left((-D)^{1 / 2}\right)$ or $f_{1}\left((-D)^{1 / 2}\right)$ from the equation for $f\left(\left(-D^{\prime}\right)^{1 / 2}\right)$ or $f_{1}\left(\left(-D^{\prime}\right)^{1 / 2}\right)$ by means of the so-called Schläfli's modular equation** of order $s$ as

$\dagger$ Comptes Rendus, vol. 48 (1859), pp. 1101-1102; vol. 49 (1860), p. 18.

$\ddagger$ Monatsberichte, Akademie der Wissenschaften, Berlin, 1863, p. 44.

$\S$ Modular equations and approximations to $\pi$, Quarterly Journal of Mathematics, vol. 45 (1914), p. 354. Collected Papers, pp. 25-26.

|| Elliptische Functionen und Algebraische Zahlen, Braunschweig, 1891.

If Weber, loc. cit., pp. 499-504. Ramanujan, Quarterly Journal of Mathematics, vol. 45 (1914), pp. 350-372; Collected Papers, pp. 23-39. G. N. Watson, Quarterly Journal of Mathematics, vol. 3 (1932), pp. 81-98 and 189-212; Proceedings of the London Mathematical Society, (2), vol. 40 (1936), pp. 83-142; Acta Arithmetica, vol. 1 (1936), pp. 284-323; Proceedings of the London Mathematical Society, (2), vol. 42 (1937), p. 377.

** Schläfli, Journal für Mathematik, vol. 72 (1870), pp. 360-369; Weber, loc. cit., pp. 267-274; Berry, American Journal of Mathematics, vol. 30 (1908), pp. 156-159; Watson, Journal für Mathematik, vol. 169 (1933), pp. 238-251. 
illustrated below. These transformation equations have been worked out for the first dozen prime values of $s$ as well as for $s=9,25$, and 49 . For our purposes the most important cases are $s=5,7,11,25$, and 49 .

The general plan is then as follows. Given $n$ such that $24 n-1$ contains $q^{\alpha}$, select a divisor $D$ of $24 n-1$ such that $(24 n-1) / D$ is a square $\geqq 1$. Also $D$ should be chosen large enough to make the infinite products (3) and (4) rapidly convergent and small enough so that either $f\left((-D)^{1 / 2}\right)$ or $f_{1}\left((-D)^{1 / 2}\right)$ is readily found either from tables or from one or more applications of Schläfli's modular equation of order $p$ or $p^{2}$ to equations already tabulated. Having found this equation we solve it approximately to as many significant figures as are required of $e^{\mu}$. Knowing the left member of either (3) or (4), we easily solve for exp $\left[\pi D^{1 / 2} / 24\right]$, an integer power of which is $e^{\mu}$.

We illustrate the procedure with the example of $n=14031$; in this case $24 n-1=11^{4} \cdot 23$. Selecting $D=11^{2} \cdot 23$, we first find the modular equation for $f\left(11(-23)^{1 / 2}\right)$. We start however with $f\left((-23)^{1 / 2}\right)$. If we set

$$
f\left((-23)^{1 / 2}\right)=2^{1 / 2} x
$$

then $\dagger$

$$
x^{3}-x-1=0
$$

If now we set

$$
f\left(11(-23)^{1 / 2}\right)=2^{1 / 2} y, \quad A=\left(\frac{x}{y}\right)^{6}+\left(\frac{y}{x}\right)^{6}, \quad B=2 x y-\frac{1}{x y},
$$

then the Schläfli modular equation of the 11 th order is $\ddagger$

$$
A-B^{5}+B^{3}+2 B=0 \text {, }
$$

or, in terms of $x$ and $y$,

$$
\begin{gathered}
y^{12}-32(x y)^{11}+88(x y)^{9}-88(x y)^{7}+44(x y)^{5} \\
-11(x y)^{3}+x y+x^{12}=0 .
\end{gathered}
$$

To obtain the equation satisfied by $y$, we must eliminate $x$ between (6) and (7). The easiest way to do this is to write (7) with $x=x_{1}, x_{2}, x_{3}$, where these are approximate roots of (6), and to multiply these equations together. Although only approximate values of $x$ are used, the exact integral coefficients of the various powers of $y$ are unmistakably recognized. Thus we readily find that $y=f\left(11(-23)^{1 / 2}\right) / 2^{1 / 2}$ satisfies

$\dagger$ Weber, loc. cit., p. 500.

$\ddagger$ Weber, loc. cit., p. 273. 
(8)

$$
\begin{aligned}
y^{36} & -704 y^{35}-1024 y^{34}-31712 y^{33}+36608 y^{32}+89496 y^{31} \\
& -65472 y^{30}-225060 y^{29}+18304 y^{28}+343519 y^{27}+32384 y^{26} \\
& -366080 y^{25}-29051 y^{24}+316096 y^{23}+10120 y^{22}-236808 y^{21} \\
& -4356 y^{20}+156816 y^{19}+17622 y^{18}-91080 y^{17}-17578 y^{16} \\
& +47047 y^{15}+8095 y^{14}-18975 y^{13}-230 y^{12}+5752 y^{11}-1111 y^{9} \\
& -176 y^{8}-143 y^{7}+396 y^{6}-66 y^{5}-55 y^{4}+78 y^{3}+22 y^{2} \\
& -y+1=0 .
\end{aligned}
$$

A preliminary reconnaissance shows that the largest root is more than 100 times as large as the absolute value of any other root, so the root squaring method of Dandelin and Graeffe is an especially good method to use in finding this largest root. Moreover, it is not with $y$ but rather with $y^{4}, y^{8}, y^{16}$, and $y^{32}$ that we are concerned, as the following argument shows. In fact, since $\mu=\pi 11^{2}(23)^{1 / 2} / 6$, we obtain from (8), with $D=11^{2} \cdot 23$,

$$
\begin{aligned}
& f^{4}\left(11(-23)^{1 / 2}\right)=4 y^{4} \\
& \quad=e^{\mu / 11}\left(1+\exp \left[-11 \pi(23)^{1 / 2}\right]\right)^{4}\left(1+\exp \left[-33 \pi(23)^{1 / 2}\right]\right)^{4} \ldots
\end{aligned}
$$

from which

$$
\begin{aligned}
e^{\mu} & =\left(4 y^{4}\right)^{11}\left(1+\exp \left[-11 \pi(23)^{1 / 2}\right]\right)^{-44} \cdots \\
& =4^{11} y^{44}\left(1-44 \exp \left[-11 \pi(23)^{1 / 2}\right]+\cdots\right) .
\end{aligned}
$$

Therefore

$$
\exp \left[-11 \pi(23)^{1 / 2}\right]=e^{-6 \mu / 11}=4^{-6} y^{-24}+\cdots .
$$

Substituting this in (9) we have

$$
e^{\mu}=4^{11} y^{44}-44 \cdot 4^{5} y^{20}+\cdots
$$

or

$$
e^{\mu}=4194304 y^{4} y^{8} y^{32}-45056 y^{4} y^{16}+\cdots \cdot
$$

Now the sum of the 128 th powers of the roots of (8) differs from $y^{128}$ by a negligible amount. Applying the root squaring method 7 times (retaining only such coefficients as are required), we find $S_{128}$, and by taking successive square roots we obtain the powers of $y$ needed in (10). Thus we find

$$
\begin{array}{rlllllllll}
e^{\mu}=90 & 55985 & 87059 & 70450 & 84877 & 28959 & 00188 & 79038 & 39298 & 26173 \\
19183 & 33361 & 28658 & 25306 & 34561 & 94199 & 90989 & 58100 & 30392 \\
& 77156 & 94755 & 49177 & 19559 & 23783 & 93967 & 06869 & 92162.896 .
\end{array}
$$


In conclusion we give the results of applying the Hardy-Ramanujan series for $p(n)$ when $n=1224,2052,2474$, and 14031. If we denote by $S_{n}(N)$ the sum of the first $N$ terms of the series, so that

$$
p(n)=S_{n}(N)+r_{n}(N)
$$

we find that

$$
\begin{array}{llllllll}
S_{1224}(16)=1 & 09765 & 80560 & 41816 & 32042 & 44403 & 44269 & 05625.00262, \\
S_{2052}(19)=20 & 25018 & 07589 & 97203 & 96101 & 85629 & 65051 & 51746 \\
& 73735 & 63574.00612, & & & \\
S_{2474}(26)=14 & 86398 & 21708 & 90271 & 21199 & 41978 & 56277 & 70438 \\
& 51222 & 84071 & 75000.00325, & & & \\
S_{14031}(62)=92 & 85303 & 04759 & 09931 & 69434 & 85156 & 67127 & 75089 \\
& 29160 & 56358 & 46500 & 54568 & 28164 & 58081 & 50403 \\
& 46756 & 75123 & 95895 & 59113 & 47418 & 88383 & 22063 \\
43272 & 91599 & 91345 & 00745.00016 . & &
\end{array}
$$

If we take the greatest integers in these $S$ 's, it is found that the resulting numbers are divisible by the proper powers of 5 and 11 to verify Ramanujan's conjecture. Since this conjecture has been proved for $q^{\alpha}=11^{2}$ and $5^{3}$, it follows that these greatest integers are either true values of the partition function or else differ from such values by multiples of 121 or 125 . It follows from (2), however, that this second alternative is impossible since the values of $\left|r_{n}(N)\right|$ are in all cases found to be less than unity. We have therefore established the following facts, all of which are in accord with the conjecture of Ramanujan :

$$
\begin{aligned}
& p(1224) \text { is divisible by } 5^{4}, \\
& p(2052) \text { is divisible by } 11^{3}, \\
& p(2474) \text { is divisible by } 5^{5}, \\
& p(14031) \text { is divisible by } 11^{4}
\end{aligned}
$$

In no case is the number of partitions divisible by a higher power of 5 or 11 than that indicated.

LeHIGH UNIVERSITY 